\title{
Clinical Outcome of Patients with Acute Inferior Wall Myocardial Infarction: Comparative Study between Left Circumflex Artery and Right Coronary Artery as Aculprit Artery \\ M.M.Ali, M.Abdel-Shafy.Tabl, H.Kh.Rashid and A.M.Abosrea \\ Cardiology Dept., Faculty of Medicine, Benha Univ., , and National Heart Institute, Egypt \\ E-Mail: amrabosrea5@gmail.com
}

\begin{abstract}
Information available about comparison between inferior wall myocardial infarction (MI) caused by RCA and LCX occlusion is limited. The aim of present study was to compare the difference between outcome of Left Circumflex artery and Right Coronary artery related acute inferior wall myocardial infarction undergoing emergency percutaneous coronary intervention. This prospective, observational, nonrandomized study enrolled 200 consecutive patients with inferior wall STEMI. The study was done at the National Heart Institute(NHI) and Benha university hospital (BUH),Cairo, Egypt in the period from January 2018 to March 2020.All patients were treated with emergency percutaneous coronary intervention during hospitalization and clinical characteristics and outcomes were compared .Group 1 included 100 patients presented with acute inferior wall STEMI caused by RCA occlusion and Group 2 included 100 patients presented with acute inferior wall STEMI caused by LCX occlusion .In hospital mortality, reinfarction, bleeding and stroke were reported in all patients.Total primary outcome in the present study was higher in $\operatorname{LCX}$ group $(\mathrm{p}=0.048)$ that may be related to higher use stents. Heart failure, stroke and bleeding were more than RCA group. More cardiac enzyme release in LCX group $(\mathrm{p}=0.046)$,also regard the 30 days outcome, the current study shows a higher incidence of combined end point of adverse cardiovascular events (Death and re-infarction) in LCX group compared to RCA group but not reaching statistically significance. Conclusions: The results of the current study suggest that LCX related acute inferior STEMI had an unfavorable clinical outcome after emergency PCI compared to RCA related acute inferior STEMI mostly related to relative increase in major or minor bleeding, stroke and reinfarction.
\end{abstract}

\section{Introduction}

Acute myocardial infarction (MI) is caused by plaque rupture in one of epicardial coronary arteries [1] clinical outcome between anterior and inferior wall MI has been extensively investigated. Nienhuis et al [2], showed more unfavorable short and longterm clinical outcomes for anterior compared to inferior MI. The size of myocardial damage in acute left anterior descending artery (LAD) occlusion is commonly big than in either acute right coronary (RCA) or left circumflex (LCX) artery occlusion because it perfuses a larger myocardial area. However, some spesific electrocardiographic features, including right ventricular infarction, reciprocal ST-segment depression in chest leads, or complete heart block, have been reported to contribute adversely to the prognostic outcome in patients presented with inferior wall MI [3] and so, patients presented with inferior wall MI do not always have favorable clinical outcomes [4].

information available about comparison between inferior wall myocardial infarction (MI) caused by RCA and LCX occlusion is limited. It has often been stated that patients with RCA related acute inferior wall MI might have a unfavorable clinical outcomes because of the higher frequency of rhythm and conduction disturbances [5]. On the other hand, the prognostic clinical outcomes of LCX- related MI is less clear. In large randomized trials on ST-segment elevation myocardial infarction (STEMI) less than $20 \%$ of cases had LCX as a culprit artery, many cases not reported due to limited or absent ST-segment elevation [6].
Rasoul et al [7], showed that enzymatic infarct size was significantly lower and Left ventricular ejection fraction (LVEF) significantly higher in patients with RCA- related MI treated by primary percutaneous intervention (PCI) when compared to LCX- related MI. Also Chen [8] and his colleagues studied 646 patients with ST-segment elevation inferior wall $\mathrm{MI}$ and showed that $\mathrm{LCx}$-related infarcts carry less favorable 30-day prognostic outcome. These findings raised our interest to test the hypothesis that the clinical outcomes of acute inferior wall MI caused by RCA and LCX occlusion may be different for patients undergoing emergency percutaneous coronary intervention (PCI).

\section{Patients and methods Study Design}

This prospective, observational, nonrandomized study enrolled 200 consecutive patients with inferior wall STEMI .The study was done at the National Heart Institute (NHI) and Benha university hospital(BUH),Cairo, Egypt in the period from January 2018 to March 2020.All patients were treated with emergency percutaneous coronary intervention. All patients signed an informed consent and the study was approved by the local ethics committee .Key inclusion criteria were: Patients who were presented within 12 hours from the onset of symptoms (characteristic chest pain lasting for at least 30 minutes, not responsive to nitrates), with a new, or presumed new ST segment elevation in 2 or more contiguous leads of at least $1 \mathrm{~mm}$ at the $\mathrm{J}$ point in inferior leads (II-III-avf) undergoing emergency 
PCI. Exclusion criteria were: Rescue PCI, cardiogenic shock and terminal liver disease.

\section{Methods}

\subsection{Baseline evaluation}

All pateints had review of their medical history on admission to emergency department including analysis of demographic data (age, sex), presence of risk factors of CAD (smoking, diabetes, hypertension, dyslipidemia and family history of ischemic heart disease), history of previous ischemic attacks or coronary revascularization( PCI or CABG),history of comorbidities and time of onset of chest pain ( $<3$ hours or 3 to 6 hours or more than 6 hours),general and cardiac examination, 12 leads ECG which was performed immediately on admission and every 6 hours during the first 24 hours, and once daily untile discharge, routine laboratory investigations including cardiac biomarkers.

\subsection{Coronary angiography and emergency PCI}

Aspirin (300 mg loading ,then $75 \mathrm{mg}$ maintenance) and clopidogrel (600 mg loading, then $150 \mathrm{mg} /$ day maintenance for one week, then 75 $\mathrm{mg} /$ day for one year) were given on admission and after emergency PCI. Un-fractionated heparin (UFH) of 10000 units bolus dose was given after sheath insertion. The procedure was done according to the standard techinique for coronary angiography and PCI. Transfemoral approach was done in all patients by using 6 Fr sheaths. Diagnostic coronary angiography was done to explor non-infarct related artery. XB or Judkin left guide catheters were used during emergency PCI in left system, while Judkin right catheter in RCA .Aspiration catheters were used in lesions with heavy thrombus burden and or impaired TIMI flow after emergency PCI. Drug eluting stents were used in all patients. The operator determined the size, length of the stent. Sheaths were removed 4-6 hours after the procedure or 4 hours after stop of GPI infusion. PCI of culprit vessel only was done.

\subsection{Study end points}

a) Primary end point: Composite end point of inhospital adverse cardiovascular events (death , reinfarction, stent thrombosis, bleeding (according to TIMI classification), heart failure and stroke.

b) Secondary end point : 30 days all cause mortality and reinfarction.

\subsection{Statistical analysis}

Data were collected and analyzed by SPSS (version 25). Data were presented as mean+ SD for continous data and as number $(\%)$ for categorial data. Between groups comparison was done using student t-test for continous data and by Chi-square test (or Fischer exact test) for qualitative data. Level of evidence was detected to be significant at $\mathrm{P}$ value $<0.05$.

\section{I- Study population}

The mean age was 59.2 years $(60.6$ years versus 57.8 years in group 1 and 2 respectively, $\mathrm{P}=0.200), 71 \%$ were males ( $62 \%$ versus $80 \%$ in group 1 and 2 respectively, $\mathrm{P}=0.005), 33 \%$ had diabetes (34\% versus $32 \%$ in group 1 and 2 respectively $\mathrm{P}=0.764), 51 \%$ had hypertension (52\%versus $50 \%$ in group 1 and 2 respectively $\mathrm{P}=0.777), 32 \%$ had dyslipidemia(30\% versus $34 \%$ in group 1 and 2 respectively $\mathrm{P}=0.544), 47 \%$ were smokers $(42 \%$ versus $52 \%$ in group 1 and 2 respectively $\mathrm{P}=0.157$ ), $24 \%$ had positive family history of CAD (20\% versus $28 \%$ in group 1,2 respectively $\mathrm{P}=0.199$ ).

Nineteen $\%$ of patients had prior history of MI ( $18 \%$ versus $20 \%$ in group 1,2 respectively $\mathrm{P}=718$ ), $16 \%$ had history of prior PCI (16\% versus $16 \%$ in group 1,2 respectively $\mathrm{P}=1.000$ ), no history of prior $\mathrm{CABG}$ in both group. Between groups comparison showed statistical significant difference between group (1) and group (2) regarding sex while no statistically significant difference was found between them regarding other baseline characteristics Table (1).

Table (1) Baseline characteristics of study population .

\begin{tabular}{|c|c|c|c|c|c|}
\hline \multirow[b]{3}{*}{ Age (years), mean (SD) } & \multirow{2}{*}{\multicolumn{2}{|c|}{$\frac{\operatorname{RCA}(\mathbf{n}=100)}{\mathrm{n}(\%)}$}} & \multirow{2}{*}{\multicolumn{2}{|c|}{$\frac{\mathrm{LCX}(\mathrm{n}=100)}{\mathrm{n}(\%)}$}} & \multirow{3}{*}{$\frac{\text { p-value }}{0.200}$} \\
\hline & & & & & \\
\hline & 60.6 & $(9.5)$ & 57.8 & $(7.8)$ & \\
\hline Sex (Male) & 62 & $(62.0)$ & 80 & $(80.0)$ & 0.005 \\
\hline Diabetes & 34 & $(34.0)$ & 32 & $(32.0)$ & 0.764 \\
\hline Hypertension & 52 & $(52.0)$ & 50 & $(50.0)$ & 0.777 \\
\hline Family history & 20 & $(20.2)$ & 28 & $(28.0)$ & 0.199 \\
\hline Smoking & 42 & $(42.0)$ & 52 & $(52.0)$ & 0.157 \\
\hline Dyslipidemia & 30 & $(30.0)$ & 34 & $(34.0)$ & 0.544 \\
\hline Prior MI & 18 & $(18.0)$ & 20 & $(20.0)$ & 0.718 \\
\hline Prior PCI & 16 & $(16.0)$ & 16 & (16.0) & 1.000 \\
\hline Prior GABG & 0 & $(00.0)$ & 0 & $(00.0)$ & - \\
\hline
\end{tabular}




\section{II- Time from onset of symptoms to admission}

Time from onset of symptoms to hospital admission was less than 3 hours in $30 \%$ of patient in group 1, versus $36 \%$ hours in group 2, pateints presented within 3 to 6 hours were $36 \%$ versus $28 \%$ in group 1, 2, while time was more than 6 hours in $34 \%$ in group 1 versus $36 \%$ in group $2, \mathrm{P}=0.449$.

\section{III- Clinical presentation on admission}

Chest pain was the main symptoms on admission in both groups $(100 \%), 24 \%$ of patients were presented with dyspnea (26\% in group 1 versus $22 \%$ in group $2, \mathrm{P}=0.508), 18 \%$ of patients were presented with palpitations (12\% in group 1 versus $24 \%$ in group $2, \mathrm{P}=0.027$ ) no significant difference was found between both groups except for palpitation.

The mean heart rate was $96.27 \pm 19.18 \mathrm{bpm}(88.0$ versus $101.3 \mathrm{bpm}$ in group 1,2 respectively, $P$ $=0 .<0.001)$, the mean SBP was $143.02+22.43 \mathrm{mmHg}$ (149.7 $\mathrm{mmHg}$ versus $147.2 \mathrm{mmHg}$ in group 1,2 respectively, $\mathrm{P}=0.449$ ), the mean DBP was $87.6 \mathrm{mmHg}(87.6 \mathrm{mmHg}$ versus $89.6 \mathrm{mmHg}$, in group 1,2 respectively, $\mathrm{P}=0.338$ ), heart block was found in 9 patients versus 2 patients in group 1,2 respectively , $\mathrm{p}=0.030$, heart block and heart rate were significantly different between the two groups .

\section{IV- Laboratory and echocardiographic finding}

The mean creatinine level was 1.0 in group 1 versus 0.9 in group $2, \mathrm{P}=0.271$. The mean ejection fraction was 48.1 versus 48.2 in group 1 and 2 , respectively, $\mathrm{P}=0.931$. Diastolic dysfunction was found in $60 \%$ and $48 \%$ in group 1 and 2 respectively, $\mathrm{P}=0.089$.significant mitral regurge was found $4 \%$ in group 1 versus $7 \%$ in group 2 respectively, $p=0.352$.

Table (2) Procedural data.
Mean cardiac enzymes level was significantly higher in group 2 than group $1, \mathrm{P}=0.046$.

\section{V-Procedural data}

All patients received 10000 units of unfractionated heparin (UFH) pre PCI, femoral approach was done in all patients using 6 fr. sheath, XB guiding catheter was used in $15 \%$ of group 2 patients and JR was us in $85 \%$ in group 1 patients, while in $75 \%$ of patients $\mathrm{JL}$ were used in group 2, floppy wire was used in $91 \%$ of all patients, while covered wire in $9 \%$ of patients $(\mathrm{p}=0.138)$.

The stent number was one in $72 \%$ of all patients (66\% versus $78 \%$ in group 1,2 respectively ), while multiple stents in $28 \%$ of all patients $(34 \%$ versus $22 \%$ in group 1,2 respectively) $\mathrm{P}=0.059$,TIMI flow after emergency PCI was III in $87 \%$ of all patients ( $86 \%$ versus $88 \%$ in group 1,2 respectively), TIMI flow II was $10 \%$ of all patients $(12 \%$ versus $8 \%$ in group 1,2 respectively), while TIMI flow I was $3 \%$ of all patients (2\% versus $4 \%$ in group 1,2 respectively) $\mathrm{P}=0.742$. the mean procedural time was $47.28+6.30$ minutes in all patients $(47.78 \pm 6.48 \mathrm{~min}$ versus $46.78 \pm 6.14 \mathrm{~min}$ in group 1,2 respectively, $\mathrm{P}$ $=0 . r(0)$, the procedure related complications as the no reflow occurred in $3 \%$ of all patients $(4 \%$ versus $2 \%$ of group 1,2 respectively, $\mathrm{P}=0.687$ ), dissection occurred in $3 \%$ of all patients( $2 \%$ versus $4 \%$ of group $1,2$ respectively, $\mathrm{P}=0.687)$. $\mathrm{RCA}$ was dominant in $58 \%$ in group 1 versus $55 \%$ in group 2 , $\mathrm{P}=0.066$.Aspiration device was used in $8 \%$ versus $18 \%$ in group 1,2 respectively ( $\mathrm{P}=0.036$ ). GP IIb/IIIa inhibitors was used in $24 \%$ versus $37 \%$ in group 1,2 respectively $(\mathrm{P}=0.046)$. Total dose of heparin was used more in group 2 than group $1(\mathrm{P}=0.042)$ Table (2)

\begin{tabular}{|c|c|c|c|c|c|}
\hline & \multicolumn{2}{|c|}{$\operatorname{RCA}(n=100)$} & \multicolumn{2}{|c|}{$\operatorname{LCX}(n=100)$} & \multirow[b]{2}{*}{ p-value } \\
\hline & $\mathbf{n}$ & $(\%)$ & $\mathbf{n}$ & $(\%)$ & \\
\hline$\overline{\text { PCI time /min, mean (SD) }}$ & 47.78 & $(6.48)$ & 46.78 & $(6.14)$ & 0.320 \\
\hline \multicolumn{6}{|l|}{ Number of diseased vessels } \\
\hline Single V. disease & 46 & $(46.0)$ & 39 & $(40.0)$ & \multirow{3}{*}{0.485} \\
\hline Two V. disease & 36 & $(36.0)$ & 37 & $(36.0)$ & \\
\hline Three V. disease & 18 & $(18.0)$ & 24 & $(22.0)$ & \\
\hline \multicolumn{6}{|l|}{ Guiding wire } \\
\hline Floppy & 94 & $(94.0)$ & 88 & $(88.0)$ & \multirow[t]{2}{*}{0.138} \\
\hline Covered & 6 & $(6.0)$ & 12 & $(12.0)$ & \\
\hline \multicolumn{6}{|l|}{ Number of stents } \\
\hline Single & 66 & $(66.0)$ & 78 & $(78.0)$ & \multirow[t]{2}{*}{0.059} \\
\hline Multiple & 34 & $(34.0)$ & 22 & $(22.0)$ & \\
\hline Reference vessel diameter, mean (SD) & 3.6 & $(0.4)$ & 3.2 & $(0.5)$ & $<0.001$ \\
\hline Lesion length, mean (SD) & 24.1 & $(6.2)$ & 20.7 & (5.3) & $<0.001$ \\
\hline \multicolumn{6}{|l|}{ TIMI flow post-PCI } \\
\hline $\mathbf{I}$ & 2 & $(2.0)$ & 4 & $(4.0)$ & \multirow{3}{*}{0.474} \\
\hline II & 12 & $(12.0)$ & 8 & $(8.0)$ & \\
\hline III & 86 & $(86.0)$ & 88 & $(88.0)$ & \\
\hline \multicolumn{6}{|l|}{ Procedural complications } \\
\hline Dissection & 4 & $(4.0)$ & 2 & (2.0) & 0.687 \\
\hline Perforation & 2 & $(2.0)$ & 1 & $(1.0)$ & - \\
\hline No reflow & 4 & $(4.0)$ & 2 & $(2.0)$ & 0.687 \\
\hline Dominant vessel & 58 & $(58.0)$ & 45 & $(45.0)$ & 0.066 \\
\hline
\end{tabular}




\begin{tabular}{|c|c|c|c|c|c|}
\hline \multicolumn{6}{|l|}{ Table (2) Continue } \\
\hline Non dominant vessel & & $42(42.0)$ & & $55(55.0)$ & 0.066 \\
\hline Aspiration device & 8 & $(8.0)$ & 18 & (18.0) & 0.036 \\
\hline GP IIb/IIIa inhibitors & 24 & $(24.0)$ & 37 & $(73.0)$ & 0.046 \\
\hline \multicolumn{6}{|l|}{ Total dose of heparin } \\
\hline 10000 iu & 78 & & 67 & & 0.042 \\
\hline $15000 \mathrm{iu}$ & 22 & & 33 & & \\
\hline
\end{tabular}

\section{VI- In hospital outcome}

Primary end point was reported in $44 \%$ of patients in group 1 versus $58 \%$ in group $2(\mathrm{p}=0.048)$. Minor bleeding occurred in $9 \%$ of all patients $(8 \%$ versus $10 \%$ in group 1 and 2 respectively, $\mathrm{P}=0.621)$. Major bleeding occurred in $2 \%$ of all patients $(1 \%$ versus $3 \%$ in group 1,2 respectively, $\mathrm{P}$ $=0.312$ ). Recurrence of chest pain was reported in $8 \%$ of all patients (8\% versus $8 \%$ of groupland 2 , respectively, $\mathrm{P}=1.000$ ). contrast induced

Table (3) In hospital outcome. nephropathy was evident in $4 \%$ in all patients $(3 \%$ versus $5 \%$ of group 1,2 respectively, $\mathrm{P}=0.470$ ), stent thrombosis occurred in $2.5 \%$ of all patients $(3 \%$ versus $2 \%$ in group 1,2 respectively, $\mathrm{P}=0.650)$, heart failure occurred in $10 \%$ of all patients (7\% versus $13 \%$ in group 1,2 respectively, $\mathrm{P}=0.157$ ), ventricular arrhythmia was reported also in $7 \%$ of all patients (9 $\%$ versus $5 \%$ in group 1,2 respectively, $\mathrm{P}=0.267$ ), death occurred in 2 patients in group 1 and 3 patients in group 2,(P=0.651). No reported cases of cardiogenic shock in either groups Table (3)

\begin{tabular}{|c|c|c|c|c|c|}
\hline \multirow[b]{3}{*}{ Primary endpoint } & \multirow{2}{*}{\multicolumn{2}{|c|}{$\begin{array}{c}\text { RCA }(n=100) \\
n(\%)\end{array}$}} & \multirow{2}{*}{\multicolumn{2}{|c|}{$\begin{array}{c}\operatorname{LCX}(n=100) \\
n(\%)\end{array}$}} & \multirow{3}{*}{$\frac{\text { p-value }}{0.048}$} \\
\hline & & & & & \\
\hline & 44 & $(44.0)$ & 58 & $(58.0)$ & \\
\hline Stent thrombosis & 3 & $(3.0)$ & 2 & $(2.0)$ & 0.650 \\
\hline Renal impairment & 3 & $(3.0)$ & 5 & $(5.0)$ & 0.470 \\
\hline Major bleeding & 1 & $(1.0)$ & 3 & $(3.0)$ & 0.312 \\
\hline Minor bleeding & 8 & $(8.0)$ & 10 & $(10.0)$ & 0.621 \\
\hline Ventricular arrhythmia & 9 & $(9.0)$ & 5 & $(5.0)$ & 0.267 \\
\hline Cardiogenic shock & 0 & $(0.0)$ & 0 & $(0.0)$ & - \\
\hline Recurrent chest pain & 8 & $(8.0)$ & 8 & $(8.0)$ & 1.000 \\
\hline Further revascularization & 2 & $(2.0)$ & 4 & $(4.0)$ & - \\
\hline Heart failure & 7 & $(7.0)$ & 13 & $(13.0)$ & 0.157 \\
\hline Stroke & 0 & $(0.0)$ & 2 & $(2.0)$ & - \\
\hline Re-infarction & 1 & $(1.0)$ & 3 & $(3.0)$ & - \\
\hline Death & 2 & $(2.0)$ & 3 & $(3.0)$ & 0.651 \\
\hline Secondary endpoint & 5 & $(5.0)$ & 10 & $(10.0)$ & 0.179 \\
\hline 30-day Mortality & 3 & $(3.0)$ & 7 & $(7.0)$ & 0.194 \\
\hline Re-infarction & 2 & $(2.0)$ & 3 & $(3.0)$ & 0.651 \\
\hline
\end{tabular}

\section{VII- 30 days outcome}

Combined end point of adverse cardiovascular events (mortality and reinfection) was reported in 7.5 $\%$ of all patients (5\% versus $10 \%$ in group 1,2 respectively $=0.460)$. All-cause mortality occurred in $5 \%$ of patients (3\% versus $7 \%$ in group 1,2 respectively, $\mathrm{P}=0.194)$. Re-infarction was reported in $2.5 \%$ of patients $(2 \%$ versus $3 \%$ in group 1,2 respectively, $\mathrm{P}=0.651$ ).

\section{Discussion}

Information available about comparison between inferior wall myocardial infarction (MI) caused by RCA and LCX occlusion is limited. It has often been stated that patients with RCA related acute inferior wall MI might have a unfavorable clinical outcomes because of the higher frequency of rhythm and conduction disturbances [5]. On the other hand, the prognostic clinical outcomes of LCX- related MI is less clear. In large randomized trials on ST-segment elevation myocardial infarction (STEMI) less than
$20 \%$ of cases had LCX as a culprit artery, many cases not reported due to limited or absent ST-segment elevation [6].

Rasoul et al [7], showed that enzymatic infarct size was significantly lower and Left ventricular ejection fraction (LVEF) significantly higher in patients with RCA- related MI treated by primary percutaneous intervention (PCI) when compared to LCX- related MI. Also Chen [8] and his colleagues studied 646 patients with ST-segment elevation inferior wall $\mathrm{MI}$ and showed that $\mathrm{LCx}$-related infarcts carry less favorable 30-day prognostic outcome. These findings raised our interest to test the hypothesis that the clinical outcomes of acute inferior wall MI caused by RCA and LCX occlusion may be different for patients undergoing emergency percutaneous coronary intervention (PCI).

We reported a mean age of 59.2 years $(60.6$ years versus 57.8 years in group 1,2 respectively, $\mathrm{P}=0.200$ ) , without significant difference between the two 
groups . Sohrabi et al., 2014 [9] evaluated the difference between outcome of left circumflex artery and right coronary artery related acute inferior wall myocardial infarction in patients undergoing adjunctive angioplasty after fibrinolysis, the mean age was $55.8 \pm 10.5$ years ' In Kim et al., 2011[10] they study Clinical outcomes of acute myocardial infarction with occluded left circumflex artery Mean age was $59.7 \pm 13.3$ years.

But we reported differences between two groups in sex, $(\mathrm{P}=0.005)$. Our study findings are in agreement with Entezarjou et al ., 2018 [11] $(\mathrm{P}<0.001)$. Entezarjou ${ }^{(11)}$ and his colleages reported a relatively higher proportion of women were observed in the RCA group as our study(38\%female

in RCA group to $20 \%$ female in LCX group \&also this difference was discordant with Chen YL., 2011[8], Sohrabi B., 2014 [9] and Rasoul S., 2007 [7] as there was no significant difference between their groups regarding sex, different study population may explain these findings.

We reported heart rate at presentation was lowest among RCA patients $(\mathrm{p}<0.001)$, The lowest median blood pressures were also found in the RCA group .Entezarjou [11] and his colleagues reported the same results.

In the present study, the mean PCI time was ( $47.78 \pm 6.48 \mathrm{~min})$ in RCA group versus $(46.78 \pm 6.14$ min)in in LCX group $(p=0.430)$. Chen YL et al., 2011[8], reported that in RCA group the mean PCI time was $41.0 \pm 14.3 \mathrm{~min}$ and the mean PCI time for LCX group was $42.0 \pm 14.9 \mathrm{~min}$. We observed rates of normal myocardial perfusion (TIMI 3 flow in $87 \%$ of patients after emergency PCI )with no significant difference between two groups $(\mathrm{P}=0.474)$. Also Chen YL et al., [8] reported TIMI 3 flow in $91.9 \%$ of RCA group and $92.9 \%$ of LCX group $(p=0.727)$. As both groups had nearly similar pre-PCI and procedural charachteristics, so the effects of these characters on the outcome in both groups also nearly similar.

In current study, major and minor bleeding were reported in group 2 (LCX group) more than group 1(RCA group), respectively due to a significant higher glycoprotein IIb/IIIa inhibitors use in group 2, $(p=0.046)$. Routine use of GPI therapy in STEMI patients results in numerical but not statistically significant reduction in mortality. GPI benefits for ischemic complications of STEMI come at the expense of an increase in bleeding complications ${ }^{(12)}$. Total heparin during the procedure was also significantly higher in group $2, \mathrm{p}=0.042$ which also describe the increase in bleeding.

Stroke was reported more in LCX group due to the higher use of aspiration devices during procedure , $\mathrm{p}=0.036$. The use of manual aspiration thrombectomy in primary PCI for STEMI has declined significantly since 2011, followed publication of the large, randomized TASTE [13] and
TOTAL [14] trials, which showed the procedure to not only be ineffective for improving clinical outcomes but also associated with an increased stroke risk following PCI.

Incidence of Heart failure was higher in LCX group that may be related to reference vessel diameter that was less than RCA group, $\mathrm{p}=<0.001$. Multivessele disease was found more in LCX group, reinfarction and stents use were also more reported in LCX group.

Total primary outcome in the present study was higher in LCX group that may be related to higher use stents. Heart failure, stroke and bleeding were more than RCA group.

We observed that there is reduction in primary endpoint with RCA group than LCX group reaching statically significant value $(\mathrm{p}=0.048)$. Heart failure reported more in LCX group $(\mathrm{p}=0.157)$, with more cardiac enzyme release also in LCX group $(\mathrm{p}=0.046)$ which may reflect more infarct size Chua SK et al.,2010 [15]. Our study findings are in agreement with Rasoul S et al., 2007 [7] which was the first study to describe the difference of clinical outcomes between patients with LCX and RCA related acute myocardial infarction who are all treated with primary angioplasty, they showed that enzymatic release, infarct size is larger and clinical outcome is worse in LCX-related acute myocardial infarction. Our study findings are also in agreement with Yip et al, [3] have previously shown that patients with inferior wall MI caused by dominant LCX occlusion had an unfavorable clinical outcome, also Sohrabi B., 2014 [9] and his coworkers Univariate analysis showed that the LCX group had significantly higher cardiac enzyme release, Significant MR and HF events than the RCA group. these results disagree with Almansori M [16] who demonestrate the in-hospital outcomes were less favorable in patients with RCA-related acute inferior wall myocardial infarction when compared to LCX related infarcts, this because of the higher frequency of rhythm and conduction disturbances and cardiogenic shock represented by the need for inotropic support. This difference can be attributed to several factors including higher prevalence of risk factors as diabetes, dyslipidemia and renal impairment noticed in patients with RCA related infarcts. The RCA was dominant in about $65 \%$ of cases reflecting a larger infarct area involved by occlusion of this artery. also Kim SS [10] and his college reported Less increased cardiac enzyme and relatively preserved ejection fraction was shown in patients with LCX group.

In the present work, although there are no significant different between both groups as regard most parameters of the in hospital outcome, there is a trend toward increased incidence of minor bleeding $(10 \%$ vs $8 \%$ ) ,major bleeding (3\% vs $1 \%$ ),renal impairment( $5 \%$ vs $3 \%)$, stroke (2\% vs $0 \%)$ , death (3\% vs $2 \%)$, heart failure (14\% vs $12 \%)$ and 
total primary end point(58\% vs $44 \%$ ) in $\operatorname{group}(2)$. However, there is marginal differences as regard stent thrombosis ( $2 \%$ vs $3 \%$ ), recurrent chest pain ( $8 \%$ vs $8 \%$ )and ventricular arrhythmia( $5 \%$ vs $9 \%$ ) in group 2 and 1 respectively. So the results of the present work revealed a trend toward reduction in bleeding complications, renal impairment, heart failure, stroke\& death in RCA group in the expense of marginal increase in stent thrombosis and ventricular arrhythmia.

As regard the 30 days outcome, the current study shows a higher incidence of combined end point of adverse cardiovascular events (Death and reinfarction) in group (2) compared to group (1), however the differences did not reach statistical significance. The rate of complication was $10 \%$ in group (2) versus 5\% in group (1) Table (3).

Our study findings are in agreement with Rasoul S et al., 2007 [7] This study shows that patients with acute myocardial infarction who undergo primary angioplasty, in whom the LCX is the infarct-related vessel, have a significantly larger infarct size and a worse clinical outcome, compared with patients in whom the RCA is involved. , Sohrabi B., 2014 [9] the prognostic in patients with RCA myocardial infarction outcomes were more favorable compared with LCX-related inferior STEMI. Chen YL et al [8], reported that LCX-related acute inferior wall myocardial infarction had significantly more incidences of advanced CHF and acute respiratory failure requiring mechanical ventilatory support. Second, the 30-day mortality rate was found lower in patients with RCA-related acute inferior wall myocardial infarction than in those with LCX-related acute inferior wall myocardial infarction. Third, the LVEF, which was found to be significantly lower in LCX-related than in RCA-related acute inferior wall myocardial infarction, was independently predictive of first month mortality.

\section{Conclusion}

The results of the current study suggest that RCA related acute inferior STEMI had a favorable clinical outcomes after emergency PCI compared to LCX related acute inferior STEMI mostly related to relative increase in major or minor bleeding, stroke and reinfarction.

\section{Recommendations}

A large prospective randomized, multi-centre trial with a longer follow up period is needed to confirm our observation.

\section{Study limitations}

- The small sample size.

- Two center study.

- Lack of randomization.

- Short period of follow up.
- Lack of proper matching of studied groups as regard baseline clinical and angiographic parameters may induce biasesults interpretation.

\section{References}

[1] M. Davies and A. Thomas, Plaque fissuring-the cause of acute myocardial infarction, sudden Ischemic death, and crescendo angina. Br Heart J, Vol.53, PP.363-73, 1985.

[2] M.Nienhuis, J.Ottervanger, J.Dambrink, Comparative predictive value of infarct location, peak $\mathrm{CK}$, and ejection fraction after primary PCI for ST elevation myocardial infarction. CoronArtery Dis, Vol.20, PP.914,2009.

[3] H.Yip, M.Chen, C.Wu, Acute myocardial infarction with simultaneous ST-segment elevation in the precordial and inferior leads: evaluation of anatomical lesions and clinical implications.Chest, Vol.123, PP.11701180,2003.

[4] H.Yip, C.Wu, M.Fu, Clinical features and outcome of patients with direct percutaneous coronary intervention for acute myocardial infarction resulting from left circumflex artery occlusion. Chest. Dec 1, Vol.122(6), PP.206874,2002.

[5] M.Fiol, I.Cygankiewicz, A.Carrillo, Value of electrocardiographic algorithm based on "ups and downs" of ST in assessment of a culprit artery in evolving inferior wall acute myocardial infarction. Am J Cardiol, Vol.94, PP.709-14, 2004.

[6] G.Stone, C.Grines, D.Cox, Controlled Abciximab and Device Investigation to Lower Late Angioplasty Complications (CADILLAC) Investigators. Comparison of angioplasty with stenting, with or without abciximab, in acute myocardial infarction. N Engl J Med, Vol.346, PP.957-66,2002.

[7] S.Rasoul, M.de Boer, H.Suryapranata , Circumflex artery-related acute myocardial infarction: limited ECG abnormalities but poor outcome. Netherlands Heart Journal. Sep 1, Vol.15(9), PP.286-90,2007.

[8] Y.Chen, C.Hang, H.Fang, Comparison of prognostic outcome between left circumflex artery-related and right coronary arteryrelated acute inferior wall myocardial infarction undergoing primary percutaneous coronary intervention. Clinical cardiology. Apr, Vol.34(4), PP.249-53,2011.

[9] B.Sohrabi, A.Separham, R.Madadi, Difference between outcome of left circumflex artery and right coronary artery related acute inferior wall myocardial infarction in patients undergoing adjunctive angioplasty after fibrinolysis. Journal of cardiovascular and thoracic research, Vol. 6(2), PP.101,2014. 
[10] S.S. Kim, H.S. Choi, M.H. Jeong, Clinical outcomes of acute myocardial infarction with occluded left circumflex artery. Journal of Cardiology. May 1, Vol.57(3), PP.290-6,2011.

[11] A.Entezarjou, M. Mohammad, P. Andell, Culprit vessel: impact on shortterm and longterm prognosis in patients with ST-elevation myocardial infarction. Open Heart, Vol.5, PP.e000852,2018.

[12] G. Wolff, A.Karathanos, L.Dannenberg, P3624 Glycoprotein IIb/IIIa inhibitor therapy in ST-segment elevation myocardial infarction: a systematic review and metaanalysis of randomized controlled trials. European Heart Journal. Aug 1, Vol.39(suppl_1), PP.ehy563-P3624,2018.
[13] S. Jolly, J. Cairns, S.Yusuf, Randomized trial of primary PCI with or without routine manual thrombectomy. N Engl J Med, Vol. 372, PP.1389-98,2015.

[14] O.Frobert, B .Lagerqvist, G.Olivecrona, Thrombus aspiration during ST-segment elevation myocardial infarction. $\mathrm{N}$ Engl J Med, Vol. 369, PP.1587-97,2013.

[15] M. Almansori and S.Ouf, Outcome comparison between left circumflex and right coronary artery-related acute inferior wall myocardial infarction. Sahel Medical Journal. Jul 1, Vol.18(3), PP.126,2015.

[16] S.Chua, H.Hung, K.Shyu, Acute ST-elevation myocardial infarction in young patients: 15 years of experience in a single center. Clinical cardiology. Mar, Vol.33(3), PP.140-8,2010. 